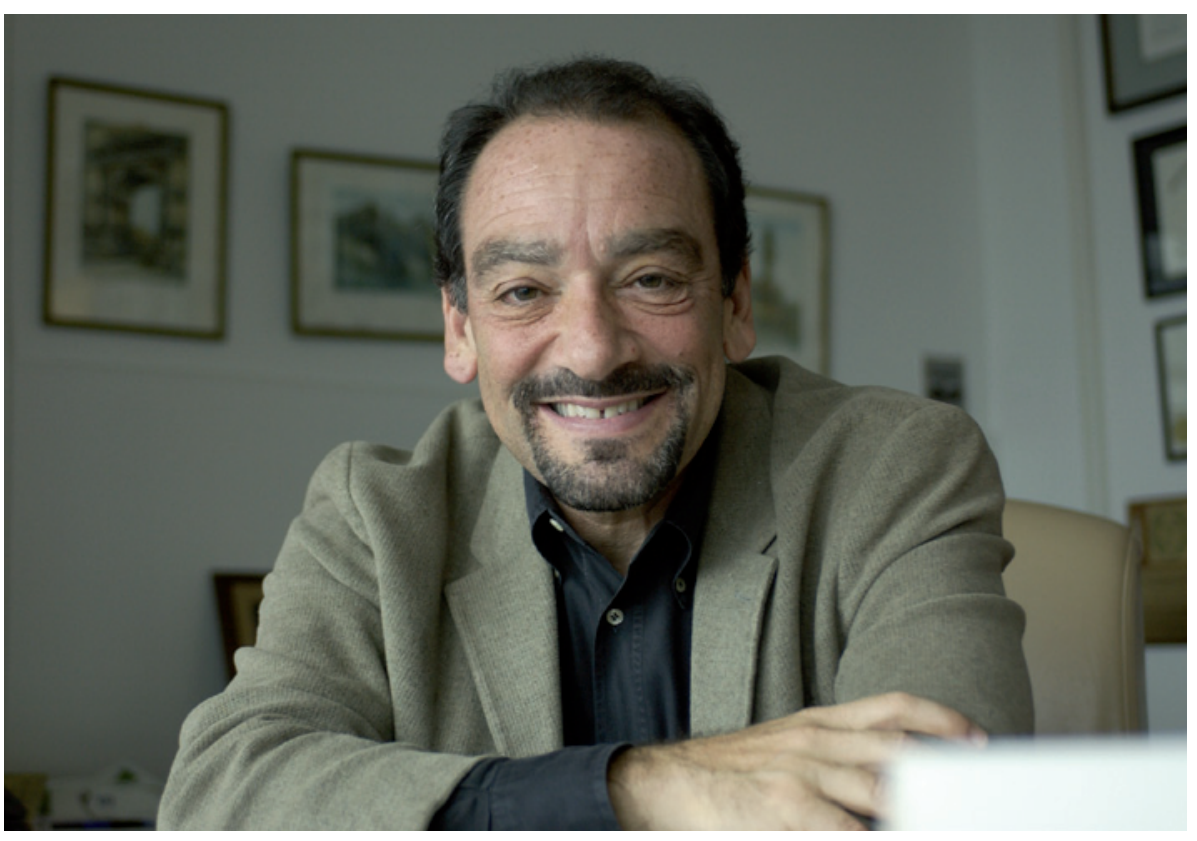

Q\&A Daniel Weinberger

\title{
A radical approach to mental illness
}

Daniel Weinberger is making a fresh start after 31 years at the National Institute of Mental Health (NIMH) in Bethesda, Maryland, where he pioneered the combined use of brain imaging and genetics in schizophrenia research. Last month, he become director of the privately funded Lieber Institute for Brain Development in Baltimore, Maryland. Founded with US\$125 million from philanthropists Stephen and Constance Lieber and Milton and Tamar Maltz, the institute's public opening is on 8 September. Weinberger talks about why it is time for new approaches to psychiatric disorders, and why he thinks his new home is the best place to pursue them.

\section{What is the Lieber Institute for Brain Development?}

It is probably the only institute in the world dedicated solely to basic and translational research on neurodevelopmental psychiatric diseases such as schizophrenia, autism spectrum disorders and bipolar disorder - all of which, we have recently understood, have their roots in abnormal brain development. Its mandate is not simply to do great basic science — although we still hope to do so - it's about changing the lives of sick people.

What limits current schizophrenia treatments? They are all based on the hypothesis that the neurotransmitter dopamine has a key role in the disease pathology. Dopamine-based therapies transformed psychiatry when they were introduced in the 1950s, because they worked well enough to allow patients to be released from hospitals. But, even though they have been greatly refined, they remain very inadequate. Some people will benefit a lot, but some won't benefit much at all. The treatments don't even affect some important symptoms of schizophrenia, such as cognitive defects.

\section{Will it be easy to move beyond this?}

Yes. I think we are poised to, thanks to genetic studies that have identified some risk genes, and to new technologies for studying the living brain and the post-mortem brain. Neuroimaging has shown us that the syndromes are associated with faulty brain circuitry. Why is the circuitry faulty? We don't understand the detailed pathology, but we've learned that risk genes - including, incidentally, some genes in dopamine biochemical pathways contribute to the aetiology by affecting the development and function of the circuitry.

All this provides us with clues to guide experiments and, as a result, the science of brain function in schizophrenia has become respectable. People who wouldn't have touched schizophrenia research with a ten-foot pole, for fear of being soiled by association with the messy biology of human behaviour - people like Ron McKay, who now leads our stem-cell programme - are now moving in.

How will the Lieber institute carry out research? We have tried to create a Google-like working environment with computer scientists, cell biologists, clinical psychiatrists and so on working together, breaking with the traditional silo-based mentality.

We'll also have a drug-development programme directed by Sol Snyder [who identified receptors for several neurotransmitters] from the Johns Hopkins School of Medicine [in Baltimore, Maryland]. Already we have four interesting new drug targets identified from our post-mortem and genetic-imaging studies. We'll try to identify additional targets by developing cellular and animal models based on the complex genetic information. It is not a question of just knocking out a gene; we'll have to model the entire molecular pathology.

We are also starting a new collection of post-mortem brains from people who had a psychiatric disorder - as well as from controls. Our brain bank will include fetal brains so we can follow the expression of risk genes at different phases of development.

Will you have external collaborations?

Absolutely. This is one of our most important aspects. We'll have partnerships with industry and with academic institutes in the United States and other countries. For example, we'll be collecting data sets to complement the huge data set I put together at the NIMH, comprising biological and genetic information from more than 3,000 patients with schizophrenia and unaffected members of their families. The new populations will be gathered by collaborators at the University of Bari in Italy and Peking University in Beijing. We'll mine all of these data sets to try to learn how risk genes contribute to the development of psychiatric disease, and also to work out if we can predict who will respond well to existing antipsychotic drugs.

\section{Couldn't you have done all this at the NIMH?}

I wish we could have done, but it wasn't possible. Our programme had a long and successful run at the NIMH. But it may be that all research programmes have a lifespan, and institutional inertia makes it difficult to keep them fresh. Another problem is that at the National Institutes of Health [the NIMH's parent agency] — despite its best intentions - it is really difficult to build outside relationships.

\section{What is the timeline for new therapies?}

We won't be able to cure schizophrenia in the next ten years, but I'm very confident that we'll have major breakthroughs by then. 